\title{
The End of Ice I
}

Daniel R. Moberg, Daniel Becker, Christoph W. Dierking, Florian Zurheide, Bernhard Bandow, Udo Buck, Arpa Hudait, Valeria Molinero, Francesco Paesani, Thomas Zeuch

Submitted date: 10/07/2019 - Posted date: 11/07/2019

Licence: CC BY-NC-ND 4.0

Citation information: Moberg, Daniel R.; Becker, Daniel; Dierking, Christoph W.; Zurheide, Florian; Bandow, Bernhard; Buck, Udo; et al. (2019): The End of Ice I. ChemRxiv. Preprint.

The appearance of ice I in the smallest possible clusters and the nature of its phase coexistence with liquid water could not thus far be unravelled. The experimental and theoretical infrared spectroscopic and free energy results of this work show the emergence of the characteristic hydrogen bonding pattern of ice I in clusters containing only around 90 water molecules. The onset of crystallization is accompanied by an increase of surface oscillator intensity with decreasing surface to volume ratio, a new spectral indicator of nanoscale crystallinity of water. In the size range from 90 to 150 water molecules, we observe mixtures of largely crystalline and purely amorphous clusters. Our analysis suggests that the liquid-ice I transition in clusters loses its sharp first-order character at the end of the crystalline size regime and occurs over a range of temperatures through heterophasic oscillations in time, a process without analog in bulk water.

File list (2)

End_of_Ice_I_main.pdf (899.42 KiB)

view on ChemRxiv - download file

The_end_of_ice_I_si.pdf (1.22 MiB)

view on ChemRxiv - download file 


\section{The End of Ice I}

Daniel R. Moberg ${ }^{1}$, Daniel Becker ${ }^{2}$, Christoph W. Dierking ${ }^{2}$, Florian Zurheide ${ }^{2}$, Bernhard Bandow, ${ }^{3}$ Udo Buck $^{4}$, Arpa Hudait, ${ }^{5}$ Valeria Molinero ${ }^{5 *}$, Francesco Paesani ${ }^{1 *}$, Thomas Zeuch ${ }^{2 *}$

${ }^{1}$ Department of Chemistry and Biochemistry, University of California, San Diego, 9500 Gilman Drive, La Jolla, California 92093, United States

${ }^{2}$ Institut für Physikalische Chemie, Universität Göttingen, Tammannstr. 6, D-37077 Göttingen, Germany

${ }^{3}$ Max-Planck-Institut für Sonnensystemforschung, Justus-von-Liebig-Weg 3, 37077 Göttingen, Germany

${ }^{4}$ Max-Planck-Institut für Dynamik und Selbstorganisation, Am Faßberg 17, D-37077 Göttingen, Germany

${ }^{5}$ Department of Chemistry, The University of Utah, Salt Lake City, Utah 84112-0850, United States

The appearance of ice $I$ in the smallest possible clusters and the nature of its phase coexistence with liquid water could not thus far be unravelled. The experimental and theoretical infrared spectroscopic and free energy results of this work show the emergence of the characteristic hydrogen bonding pattern of ice $I$ in clusters containing only around 90 water molecules. The onset of crystallization is accompanied by an increase of surface oscillator intensity with decreasing surface to volume ratio, a new spectral indicator of nanoscale crystallinity of water. In the size range from 90 to 150 water molecules, we observe mixtures of 


\section{largely crystalline and purely amorphous clusters. Our analysis suggests that the liquid-ice}

I transition in clusters loses its sharp first-order character at the end of the crystalline size regime and occurs over a range of temperatures through heterophasic oscillations in time, a process without analog in bulk water.

The emergence of macroscopic water properties and anomalies can be studied in a systematic manner in clusters. The characteristic hydrogen bond network of ice I was first observed in the 1980s in the supersonic expansion of water vapor and the critical onset size for ice I in clusters was estimated to be between 200 and 1000 water molecules ${ }^{1}$. Only in the present decade have infrared spectroscopic techniques advanced to the point where the appearance of characteristic ice I signatures can be observed with precise size resolution, first in neutral clusters with about $n=275$ molecules ${ }^{2}$ and later in $\mathrm{La}^{3+}$ doped cationic clusters at about $n=375^{3}$. These sizes represent upper limits for the onset size and, so far, could not help in distinguishing predictions of the smallest cluster that forms ice I in molecular simulations which range from above $n=123$ and below $n=293^{4}$, above $n=90^{5}$, and between $n \approx 100$ and $n=137^{6}$. Therefore, the smallest size of water clusters that crystallize into ice I on cooling has not yet been established in experiments. This situation is in contrast to the progress made since the 1990 s in unravelling the structures of smaller $\left(\mathrm{H}_{2} \mathrm{O}\right)_{n}$ clusters through size selective experiments assisted by $a b$ initio calculations ${ }^{7-11}$. The transition from two-dimensional to three-dimensional structures in $\left(\mathrm{H}_{2} \mathrm{O}\right)_{6}$ is now understood in full detail ${ }^{12-14}$ and larger individual isomers ${ }^{15}$ or cluster sizes with exceptionally stable configurations were determined in some special cases ${ }^{16-19}$. However, all these ordered isomers are specific to an individual size or a narrow size range. They are not representative of macroscopic water or ice properties. 
The large remaining uncertainty with regard to the onset of crystallization in neutral water clusters is not only due to technical limitations, like the selection of size in experiments and the accuracy of modeling predictions of cluster energies. With increasing cluster size arises a critical influence parameter in both experiment and simulation, whose importance was not sufficiently recognized in the past: the rate at which the initially supercooled liquid clusters are cooled when they begin crystallizing ${ }^{6}$. Cooling too rapidly, or insufficient residence times, can result in vitrified amorphous clusters $^{6,20}$, which explains the dependence of the observed onset size on experimental conditions like stagnation pressure in supersonic expansions ${ }^{13,21}$. A related issue is present in search algorithms for global minima structures, where strategies are developed to circumvent barriers blocking isomerization coordinates to local and global minima ${ }^{13}$. In turn, the quality of predicted energies and spectroscopic signatures of identified minima highly depends on the applied level of theory ${ }^{13}$. We can therefore state three challenges that must be considered when aiming to determine the smallest cluster size that stabilizes ice I. First, selectivity towards size and structure in the experiment; second, control of cluster dynamics and relaxation to the energetically most stable structures in experiment and molecular simulations; and third, the development of efficient water models that reliably predict both the energetics and spectroscopic features of crystalline and noncrystalline water clusters. Significant progress has been made in all three fields in recent years. In the present study we combine these advances in experiment and modeling for a comprehensive characterization of the infrared spectroscopic signatures and the thermodynamic and kinetic constraints of the emergence of ice I and the nature of its phase coexistence with liquid water in the smallest possible clusters. 
In the experiment, a molecular beam of clusters is generated in a supersonic expansion of a water-argon mixture through a conical nozzle. Such expansions result in final cluster temperatures from around $150 \mathrm{~K}$ down to about $40 \mathrm{~K}^{13}$. It only recently became possible to simulate in detail the gas and aggregation dynamics in this type of experiment for conditions enabling crystallization in water clusters consisting of around 200 water molecules ${ }^{21}$. The results of these numerical simulations and earlier experimental observations ${ }^{13}$ suggest the combination of low stagnation pressure and a slow argon seeded molecular beam for achieving low cooling rates at temperatures below $200 \mathrm{~K}$, where molecular simulations predict that crystallization occurs in clusters with less than a few hundred molecules ${ }^{4,6}$. In this work we observe the onset of crystallization in a weak expansion of 1 bar stagnation pressure and a reservoir temperature of $343 \mathrm{~K}$ that yields 0.3 mole fraction of water in argon as the carrier gas. Fig. 1 illustrates the three characteristic zones for the aggregation and gas dynamics in the conical nozzle, together with estimated temperatures and cooling rates derived from the detailed numerical simulations by Gimelshein, et. al. for a neon seeded expansion resulting in a similar size distribution ${ }^{21}$. The temperature drops rapidly in the entrance zone, establishing the size distribution. The highest cooling rates in this region approach $1 \mathrm{~K} / \mathrm{ns}$, where temperatures reach between 270 to $220 \mathrm{~K}$. The molecular beam is established at around $50 \mu \mathrm{m}$ from the nozzle entrance, where the temperatures have cooled to around $200 \mathrm{~K}$ and the cooling rates have slowed to around $0.5 \mathrm{~K} / \mathrm{ns}$. The final cluster temperatures of around $150 \mathrm{~K}$ are approached with a further decrease of gas density at around $250 \mu \mathrm{m}$, where the cooling rates have dropped below $0.1 \mathrm{~K} / \mathrm{ns}$. The validity of the final temperature estimates for this type of expansion 
has been demonstrated by tracking isomer population changes in the range of 40 to above $150 \mathrm{~K}^{13}$, see e.g. the case of the melting-like transition of the water nonamer ${ }^{22}$. The water clusters in the molecular beam are then singly tagged with $\mathrm{Na}$ atoms when passing through a pick-up cell. The sodium atoms enable their soft, fragmentation-free phototionization near the threshold of $3.2 \mathrm{eV}$ (385 nm) using UV light from a commercial dye laser. The ionized clusters are then size selectively accelerated and detected by time-of-flight mass spectrometry. The positive, size resolved IR action signal results from cluster excitation with radiation from a tunable IR laser 80 to $100 \mathrm{~ns}$ before photoionization. The action effect exploits the increased abundance of solvated $\mathrm{Na}$ atoms with low ionization energy in the heated clusters ${ }^{23}$. More details on the method are given in ${ }^{2}$ and the supplement. We use the characteristic red-shift of the absorption maximum of the hydrogen bonded $\mathrm{OH}$-stretch oscillators to detect crystalline clusters in the molecular beam ${ }^{2,3}$. Additionally, we introduce in this work a signature in the free $\mathrm{OH}$ stretch band, known for ice surfaces ${ }^{24}$, as an independent indicator for emerging crystallinity in clusters.

New simulation techniques for obtaining IR spectroscopic signals of aqueous systems complement these experimental efforts. Molecular dynamics simulations with many body potentials (MB-MD) for water have been shown to accurately reproduce structural, thermodynamic and dynamical properties of aqueous systems across both system size and phases ${ }^{25}$. In particular, the fully flexible atomistic many-body potential for water, $\mathrm{MB}-\mathrm{pol}^{26-28}$, predicts vibrational spectra that compare favorably with experimental results for systems ranging from small water clusters to the bulk phase. ${ }^{29,30}$ Here we use MB-MD simulations with the MB-pol potential energy function for water in the canonical (NVT) ensemble to equilibrate clusters, followed by MB-MD simulations in the 
microcanonical (NVE) ensemble to calculate infrared spectra from time correlation functions of the system dipole moments. This applies for all predicted cluster energies and IR spectra in this study. The initial structures of clusters with $n=70-120$ were determined with a parallel evolutionary algorithm (PEA) ${ }^{5}$, while those of crystallized clusters with $n>120$ were obtained by cooling clusters modeled with the many-body monatomic water model $\mathrm{mW}^{31}$ at the same rate as in our experiment. Following ref. ${ }^{32}$, we classify each water molecule in a cluster as either 'liquid-like' or 'ice I-like' using the CHILL+ algorithm. More details on the molecular simulation methods are given in the supplementary material.

The size resolved experimental and simulated IR spectra of clusters with about 60 to 200 water molecules are shown in Figure 2. The experiments at slow cooling conditions reveal the emergence of a shoulder in the low frequency region of the bonded (Figure 2A) and free $\mathrm{OH}$ (Figure 2B) stretch bands for clusters with $90 \pm 10$ molecules (see also Figures S1 to S3 in the supplement, where the significance of the red shifts between $n=70$ and 90 is readily apparent). The maximum intensity of the bonded $\mathrm{OH}$ stretch band for cluster sizes above $n=150$ has shifted to values below $3300 \mathrm{~cm}^{-1}$ indicating the dominance of internal $\mathrm{H}_{2} \mathrm{O}$ molecules in an 'ice I-like' configuration, as observed in previous studies ${ }^{2,3}$. The shoulder in the free $\mathrm{OH}$ stretch band has sharply increased in this size range, and there is a surprising gain in relative intensity normalized to the bonded $\mathrm{OH}$ intensity. Typically, a decrease is expected as the surface to volume ratio decreases with cluster size and, therefore, the relative number of free $\mathrm{OH}$ oscillators at the surface should decrease accordingly. This is what we observe in a control experiment (Figure 2C) at high stagnation pressure under fast cooling conditions, which produces amorphous water clusters of 
sizes up to $n=400^{13}$. In that case, the relative free $\mathrm{OH}$ stretch intensity decreases and there is no intensity gain below $3700 \mathrm{~cm}^{-1}$. A similar comparison of slow and fast cooling conditions for the bonded $\mathrm{OH}$ band also shows the divergence around $3200 \mathrm{~cm}^{-1}$ to start for clusters containing 80 to 100 water molecules (see Figure S2). We have therefore ample experimental evidence that this size range marks the lower limit for the existence of ice I in clusters. We note that the intensity gain of surface oscillators with decreasing surface to volume ratio at the onset of crystallization represents a new indicator of nanoscale water crystallinity, here observed for $n=90$ to 150 . At may also occur for larger clusters sizes ${ }^{3}$ noting that the free $\mathrm{OH}$ band was not analyzed in this respect in that study.

The early onset of crystallization in the present experiments at $90 \pm 10$ water molecules clearly supports Bandow and Hartke's prediction that water clusters with a crystalline ice I core become energetically competitive at around $n=90^{5}$. Consequently, we use the structures from their work as initial structures for simulating on MB-pol level the IR spectra in the bonded and free OH stretch regions for $n=120$ and below. We model the experimental IR spectra as arising from mixtures of crystalline and amorphous clusters, to capture the onset size of emerging crystalline signatures, which are centered around $3200 \mathrm{~cm}^{-1}$ (see Figures 2 and S1), the intensity maximum of larger purely crystalline clusters ${ }^{4}$. The simulated IR spectra of neutral clusters qualitatively capture the transition from amorphous to crystalline water clusters and the resulting red shift in the bonded IR spectrum (Figure 2D). Only amorphous clusters are used to generate the spectrum in Figure 2D at $n=70$, while ratios of amorphous to crystalline clusters of 4:1 and 1:4 were used for $n=90$ and $n=159$, respectively. This assumed shift in ratio is consistent with the trend of the simulated free 
energies in this size range, which are discussed below. The missing intensity below $3300 \mathrm{~cm}^{-1}$ (belonging to fully coordinated and single donor double acceptor (DAA) molecules) in the simulated spectra is attributed to a Fermi resonance between the $\mathrm{OH}$ stretching and $\mathrm{HOH}$ bending modes not properly captured in the simulation (32). The underpredicted bond strength of DAA oscillators may cause the missing intensity at low frequencies and may also cause the broader simulated band of the corresponding free $\mathrm{OH}$ oscillator molecules (Figures $2 \mathrm{E}, \mathrm{F}$ ). The simulations correctly predict the gain in intensity below $3700 \mathrm{~cm}^{-1}$ for the free $\mathrm{OH}$ band for slow cooling conditions (Figure 2E) that lead to crystallization and the loss of intensity above $3700 \mathrm{~cm}^{-1}$ for fast cooling conditions producing solely amorphous clusters (Figure 2F). Additionally, the simulations predict the experimentally observed gain in relative free $\mathrm{OH}$ stretch intensity when crystalline clusters are admixed to amorphous clusters. Compared to the experiment, the effect is a bit more pronounced at $n=90$ and less pronounced at $n=159$. However, quantitative agreement cannot be expected as intensities are difficult to predict and the experimental spectra are a result of not necessarily linear action spectroscopy. A very important point here is that the increase of the relative free $\mathrm{OH}$ band intensity is a strong and unambiguous effect in this size range. It is related to the presence of a crystalline core, which increases the number of surface oscillators by about $50 \%$ for $n=90$ compared to amorphous clusters as derived from the results of Bandow and Hartke ${ }^{5}$. Since the intensity evolution of the free $\mathrm{OH}$ band clearly deviates between slow and fast cooling conditions (Figure 2B and C) in the analyzed size range we can rule out that the simultaneous red shift in the bonded $\mathrm{OH}$ band, the established indicator of crystallinity in water clusters ${ }^{2,3,20}$, is related to a different structural effect. 
To rationalize the IR spectroscopic results, we analyze the relative stability of crystalline and amorphous water cluster structures, spanning from slightly below the early onset size for crystallization observed in this work ( $n=90)$ to the delayed onset sizes (up to $n=293$ ) in earlier studies $^{2,3,13}$, noting that indications for emerging crystallinity around $n=100$ were reported for protonated water clusters ${ }^{33}$. We calculate the free energies with MB-pol using the quasi-harmonic approximation $^{34,35}$ on energies and vibrational normal modes obtained from cluster structures identified by Bandow and Hartke ${ }^{5}$ as starting points for $n=70,90$, and 120 and by simulations with $\mathrm{mW}$ for all larger sizes ${ }^{6}$. Figure 3 shows the difference in free energy $F_{\text {crystalline }}-F_{\text {amorphous }}$ of optimized amorphous and crystalline clusters from $n=70$ to 293 computed at $100 \mathrm{~K}$ and $150 \mathrm{~K}$. Though there is, as expected, a temperature dependence for the amorphous to crystalline transition, we find that already for $n=90$ the crystallized and purely amorphous clusters are equally stable within the error bars at $T=100 \mathrm{~K}$ and $150 \mathrm{~K}$. We note that the discrimination between amorphous and ice-like clusters is challenging for $n=70$ due to the small crystalline core of 'ice I-like' cluster isomers and distributed molecules in 'ice I-like' geometry in amorphous clusters (see snapshots in Figure 3 and Figure S4 in the supplement). At larger cluster sizes, crystalline structures become gradually more stable and the corresponding 'ice I-like' signal is much more pronounced in the IR spectra. Snapshots of the simulated crystalline clusters in Figure 3 also illustrate the growing ice-like core in the analyzed size region. These free energy calculations are in good agreement with the GibbsThomson analysis of the size-dependent equilibrium melting temperature of water clusters, which predicts $T_{m}=140 \pm 8 \mathrm{~K}$ for $n=90$ and $T_{m}=128 \pm 9 \mathrm{~K}$ for $n=70^{6}$. Based on the indistinguishable free energies of the amorphous and crystallized clusters with $n=90$ in the simulations, the presence 
of fully crystalline (intensity increase centered around $3200 \mathrm{~cm}^{-1}$ ) and amorphous (high remaining intensity above $3400 \mathrm{~cm}^{-1}$ ) features in the experimental spectra, and the agreement between the spectra in the experiment and molecular simulations in Figure 2, we assign the experimental IR spectra above $n=90$ to mixtures of amorphous, liquid-like and crystalline clusters. The presence of mixtures is consistent with molecular heat capacities from calorimetry experiments in this size and temperature range ${ }^{36}$. The free energy calculations of Figure 3, together with the Gibbs-Thomson analysis of ref. ${ }^{6}$, paint a picture consistent with the experimental and simulated results outlined in Figure 2: the formation of a crystalline core becomes more and more energetically unfeasible below $n=100$ resulting in amorphous clusters. The disconnected nature and negligible fraction of molecules with ice I order in the $n=70$ clusters, together with the crossing between the glass transition temperature $\mathrm{T}_{g}$ and the $\mathrm{T}_{m}$ of the clusters for that size is consistent with the experimental analysis indicating that the end of ice I occurs for clusters with $n=90 \pm 10$ water molecules.

The growing stability of crystalline structures at $T=100$ and $150 \mathrm{~K}$ above the region near $n=140$ explains previous experimental assignments of onset sizes above $n=200^{2,3,13}$. This, and the coexistence of crystallized and amorphous clusters above the onset size, point to delicate thermodynamic and kinetic constraints for ice nucleation in the size range from $n=60$ to $n=300$ studied here. The interdependence of crystallization barriers and critical cooling rates was studied in simulations with the $\mathrm{mW}$ water model by Johnston and Molinero ${ }^{6}$. They found that clusters with $n$ around 400 could be crystallized at a cooling rate of $0.1 \mathrm{~K} / \mathrm{ns}$, but those with $n=159$ molecules required cooling rates slower than $0.05 \mathrm{~K} / \mathrm{ns}$ to nucleate ice at $150 \mathrm{~K}^{6}$. More intriguing, the simulations revealed that at $n=137$, the free energy barrier for the transition between all liquid and 
crystallized clusters at $150 \mathrm{~K}$ was comparable to the thermal energy, and the clusters oscillated in time between the amorphous and crystallized states with a small ice I core surrounded by disordered water ${ }^{6}$. Ice-liquid phase coexistence through oscillations has also been recently reported for water in nanoscale confinement ${ }^{37}$. Multiple heterophasic oscillations of the $n=137$ cluster occurred along hundreds of nanoseconds ${ }^{6}$, and the transitions are even faster for smaller clusters ${ }^{37}$. The assignment of the IR spectra of clusters with 90 to 150 water molecules in Figure 2 (and S1) to mixtures strongly supports that oscillations between crystallized and amorphous configurations are also present in the experiment. The presence of mixtures, together with the obvious presence of the forward reaction (liquid to crystal), supports the presence of the backward reaction under quasi thermal equilibrium conditions around $150 \mathrm{~K}$ in the beam (see Figure 1). Note that molecular heat capacities in-line with the presence of mixtures of solid and liquid clusters, although interpreted as a glassy state, were measured under equilibrium conditions in a similar size and temperature range for anionic water clusters ${ }^{36}$. These previous experiments and the observation of liquid-like and crystalline mixtures in the present study clearly support the existence of ice-liquid oscillations in water, a phenomenon first predicted three decades ago by Berry and Wales for Lennard-Jones clusters $^{38}$.

The experiments and simulations of this study give unambiguous evidence that the end of ice I occurs for clusters with $n=90 \pm 10$ water molecules through the validation and use of a new free $\mathrm{OH}$ surface indicator. Most significantly, the comprehensive analysis of this work interlinks in a consistent manner experimental and theoretical concepts for studying microscopic water properties of the past 30 years, which now can be seen in a common perspective. Just a few of the many 
examples are the links of the free $\mathrm{OH}$ band to the interior structure for clusters (this work, ref. ${ }^{5}$ ) and for bulk water ${ }^{24}$, the influence of cooling rates on onset sizes for crystallization ${ }^{6,13,21}$, and, closely related, the onset of crystallization in neutral ${ }^{1,2,13}$ and ionic clusters ${ }^{3,33}$, and the complementary character of calorimetry experiments with anionic clusters ${ }^{36}$ and the neutral cluster results of this work. Bolstered by this high degree of consistency the present study identifies the size range of $n=90 \pm 10$ water molecules as a boundary that demarcates the behavior of water clusters as "macromolecules" with multiple size-dependent isomers and the birth of the ice I phase characteristic of bulk water. Our analysis indicates further that in clusters with less than 150 water molecules the nature of the liquid-ice I transition loses its sharp first-order character and occurs over a range of temperatures through heterophasic oscillations in time. The oscillations between liquid and ice states result from the small number of molecules in the clusters and have no analog in bulk water. However, this state may exist in materials or substances like proteins that contain nanoconfined water ${ }^{37}$. Heterophasic oscillations are a new, yet hypothesized concept for nanoscale phase change dynamics that may find future applications. In general, the results of this work lend increased predictive power to state of the art molecular simulation techniques which are indispensable tools for studying the role of structures and phase transition dynamics of nanoscale water in e.g. biological, geophysical and astrophysical environments.

1. Torchet, G., Schwartz, P., Farges, J., de Feraudy, M. F. \& Raoult, B. Structure of solid water clusters formed in a free jet expansion. J. Chem. Phys. 79, 6196-6202 (1983).

2. Pradzynski, C. C., Forck, R. M., Zeuch, T., Slavíček, P. \& Buck, U. A fully size-resolved 
perspective on the crystallization of water clusters. Science 337, 1529-1532 (2012).

3. Cooper, R. J., DiTucci, M. J., Chang, T. M. \& Williams, E. R. Delayed Onset of Crystallinity in Ion-Containing Aqueous Nanodrops. J. Am. Chem. Soc. 138, 96-99 (2016).

4. Buch, V., Bauerecker, S., Devlin, J. P., Buck, U. \& Kazimirski, J. K. Solid water clusters in the size range of tens-thousands of $\mathrm{H}_{2} \mathrm{O}$ : a combined computational/spectroscopic outlook. Int. Rev. Phys. Chem. 23, 375-433 (2004).

5. Bandow, B. \& Hartke, B. Larger water clusters with edges and corners on their way to ice: Structural trends elucidated with an improved parallel evolutionary algorithm. J. Phys. Chem. A 110, 5809-5822 (2006).

6. Johnston, J. C. \& Molinero, V. Crystallization, Melting, and Structure of Water Nanoparticles at Atmospherically Relevant Temperatures. J. Am. Chem. Soc. 134, 6650-6659 (2012).

7. Liu, K. et al. Characterization of a cage form of the water hexamer. Nature 381, 501-503 (1996).

8. Cruzan, J. D. et al. Quantifying hydrogen bond cooperativity in water: VRT spectroscopy of the water tetramer. Science 271, 59-61 (1996).

9. Gruenloh, C. J. et al. Infrared Spectrum of a Molecular Ice Cube: The $\mathrm{S}_{4}$ and $\mathrm{D}_{2 d}$ Water Octamers in Benzene-(Water). Science 276, 1678-1681 (1997).

10. Buck, U., Ettischer, I., Melzer, M., Buch, V. \& Sadlej, J. Structure and Spectra of ThreeDimensional $\left(\mathrm{H}_{2} \mathrm{O}\right)_{n}$ Clusters, $n=8,9$, 10. Phys. Rev. Lett. 80, 2578-2581 (1998). 
11. Nauta, K. \& Miller, R. E. Formation of Cyclic Water Hexamer in Liquid Helium: The Smallest Piece of Ice. Science 287, 293-295 (2000).

12. Perez, C. et al. Structures of cage, prism, and book isomers of water hexamer from broadband rotational spectroscopy. Science 336, 897-901 (2012).

13. Buck, U., Pradzynski, C. C., Zeuch, T., Dieterich, J. M. \& Hartke, B. A size resolved investigation of large water clusters. Phys. Chem. Chem. Phys. 16, 6859-6871 (2014).

14. Brown, S. E. et al. Monitoring water clusters "melt" through vibrational spectroscopy. J. Am. Chem. Soc. 139, 7082-7088 (2017).

15. Pradzynski, C. C. et al. Infrared detection of $\left(\mathrm{H}_{2} \mathrm{O}\right)_{20}$ isomers of exceptional stability: a droplike and a face-sharing pentagonal prism cluster. Phys. Chem. Chem. Phys. 16, 26691-26696 (2014).

16. Lee, S.-W., Freivogel, P., Schindler, T. \& Beauchamp, J. L. Freeze-Dried Biomolecules: FTICR Studies of the Specific Solvation of Functional Groups and Clathrate Formation Observed by the Slow Evaporation of Water from Hydrated Peptides and Model Compounds in the Gas Phase. J. Am. Chem. Soc. 120, 11758-11765 (1998).

17. Ma, L., Majer, K., Chirot, F. \& von Issendorff, B. Low temperature photoelectron spectra of water cluster anions. J. Chem. Phys. 131, 144303 (2009).

18. Kazachenko, S. \& Thakkar, A. J. Water nanodroplets: Predition of five model potentials. J. Chem. Phys. 138, 194302 (2013). 
19. Zurheide, F. et al. Size-Resolved Infrared Spectroscopic Study of Structural Transitions in Sodium-Doped $\left(\mathrm{H}_{2} \mathrm{O}\right)_{n}$ Clusters Containing 10-100 Water Molecules. J. Phys. Chem. A 119, 2709-2720 (2015).

20. Manka, A. et al. Freezing water in no-mans land. Phys. Chem. Chem. Phys. 14, 45054516 (2012).

21. Gimelshein, N., Gimelshein, S., Pradzynski, C. C., Zeuch, T. \& Buck. The temperature and size distribution of large water clusters from a non-equilibrium model. J. Chem. Phys. 142, 244305 (2015).

22. Brudermann, J., Buck, U. \& Buch, V. Isomerization and melting-like transition of size-selected water nonamers. J. Phys. Chem. A 106, 453-457 (2002).

23. Dierking, C. W. et al. Revealing isomerism in sodium-water clusters: Photoionization spectra of $\mathrm{Na}\left(\mathrm{H}_{2} \mathrm{O}\right)_{n}(\mathrm{n}=2-90)$. J. Chem. Phys. 146, 244303 (2017).

24. Devlin, J. P. \& Buch, V. Surface of ice as viewed from combined spectroscopic and computer modeling studies. J. Phys. Chem. 99, 16534-16548 (1995).

25. Cisneros, G. A. et al. Modeling Molecular Interactions in Water: From Pairwise to ManyBody Potential Energy Functions. Chem. Rev. 116, 7501-7528 (2016).

26. Babin, V., Leforestier, C. \& Paesani, F. Development of a "First Principles" Water Potential with Flexible Monomers: Dimer Potential Energy Surface, VRT Spectrum, and Second Virial Coefficient. J. Chem. Theory Comput. 9, 5395-5403 (2013). 
27. Babin, V., Medders, G. R. \& Paesani, F. Development of a "first principles" water potential with flexible monomers. ii: Trimer potential energy surface, third virial coefficient, and small clusters. J. Chem. Theory Comput. 10, 1599-1607 (2014).

28. Medders, G. R., Babin, V. \& Paesani, F. Development of a "first-principles" water potential with flexible monomers. iii. liquid phase properties. J. Chem. Theory Comput. 10, 2906-2910 (2014).

29. Reddy, S. K., Moberg, D. R., Straight, S. C. \& Paesani, F. Temperature-dependent vibrational spectra and structure of liquid water from classical and quantum simulations with the mb-pol potential energy function. J. Chem. Phys. 147, 244504 (2017).

30. Moberg, D. R., Straight, S. C. \& Paesani, F. Temperature dependence of the air/water interface revealed by polarization sensitive sum-frequency generation spectroscopy. J. Phys. Chem. B 122, 4356-4365 (2018).

31. Molinero, V. \& Moore, E. B. Water modeled as an intermediate element between carbon and silicon. J. Phys. Chem. B 113, 4008-4016 (2009).

32. Nguyen, A. H. \& Molinero, V. Identification of Clathrate Hydrates, Hexagonal Ice, Cubic Ice, and Liquid Water in Simulations: the CHILL+ Algorithm. J. Phys. Chem. B 119, 9369-9376 (2015).

33. Mizuse, K., Mikami, N. \& Fujii, A. Infrared Spectra and Hydrogen-Bonded Network Structures of Large Protonated Water Clusters $\mathrm{H}^{+}\left(\mathrm{H}_{2} \mathrm{O}\right)_{n}(n=20-200)$. Angew. Chem. Intl. Ed. 49, 10119-10122 (2010). 
34. Born, M. \& Huang, K. Dynamical Theory of Crystal Lattices (Clarendon Press, Oxford, UK, 1954).

35. Carrier, P., Wentzcovitch, R. \& Tsuchiya, J. First-principles prediction of crystal structures at high temperatures using the quasiharmonic approximation. Phys. Rev. B 76, 064116 (2007).

36. C. Hock, M. Schmidt, R. Kuhnen, C. Bartels, L. Ma, H. Haberland, B. v. Issendorff. Calorimetric Observation of the Melting of Free Water Nanoparticles at Cryogenic Temperatures. Phys. Rev. Lett. 103, 073401 (2009).

37. Kastelowitz, N. \& Molinero, V. Ice-liquid oscillations in nanoconfined water. ACS Nano 12, 8234-8239 (2018).

38. R. S. Berry, D. J. Wales. Freezing, Melting, Spinodals, and Clusters. Phys. Rev. Lett. 63, 1156-1159 (1989).

Acknowledgements D.B., C.D., and F.Z. equally contributed to the experimental part of the this study. D.R.M, A.H., F.P., and VM gratefully acknowledge support by the US National Science Foundation through award CHE-1305427. T.Z. thanks the Deutsche Forschungsgemeinschaft for financial support (grants ZE890 1-2, 4-1). All calculations with the MB-pol water model used resources of the Extreme Science and Engineering Discovery Environment (XSEDE),107 which is supported by the National Science Foundation through grant ACI-1053575. A.H. and V.M. thank the Center for High Performance Computing at The University of Utah for technical support and a grant of computing time. Correspondence and requests for materials should be addressed to Valeria Molinero (email: valeria.molinero@ utah.edu), Francesco Paesani (email: fpaesani@ucsd.edu) or Thomas Zeuch (email: tzeuch1@gwdg.de). 

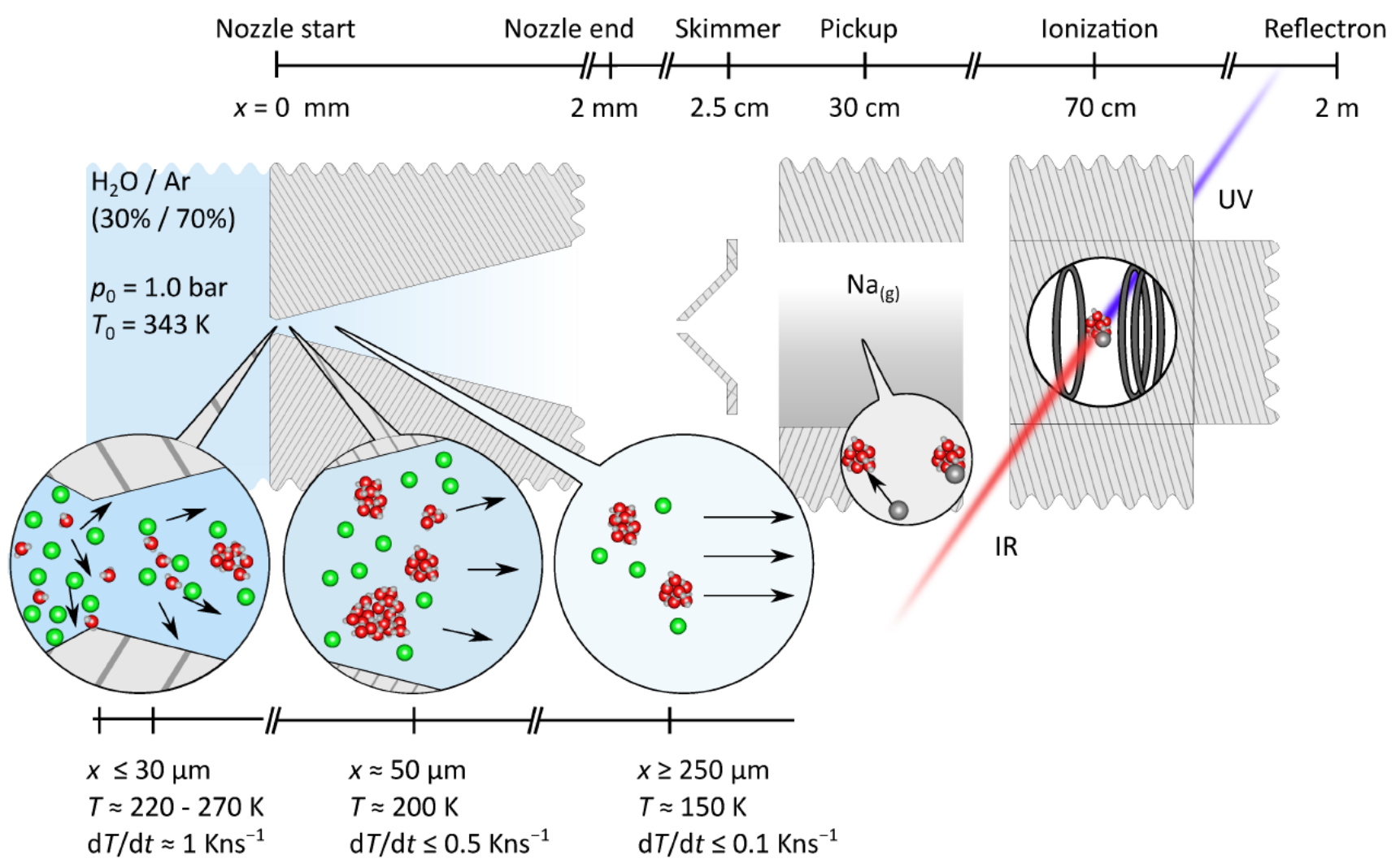

Scheme of experimental set-up with focus on aggregation dynamics in the conical nozzle. For this nozzle a detailed numerical simulation (ref. 21) has predicted the cluster size distribution, the cluster temperatures, and the cluster velocities as a function of distance from the nozzle start. From these quantities the estimate cooling rates are derived. The validity of the prediction is shown in its ability to capture the final size distribution and its shift by initial temperature variation (ref. 21). The estimated final temperature is around $150 \mathrm{~K}$. More details on the simulation and the temperature approximation are given in the supplement. 
Slow Cooling

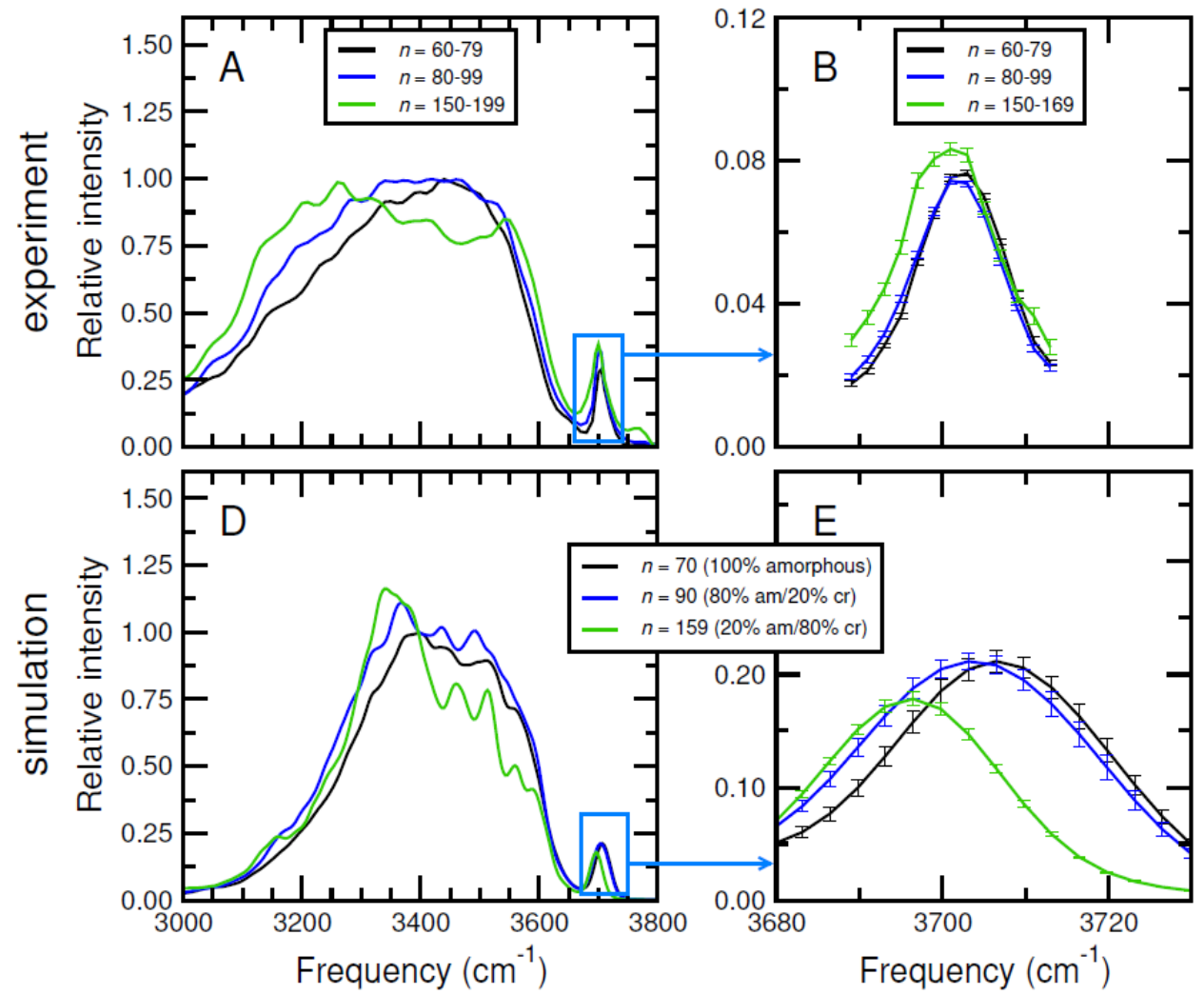

Fast Cooling

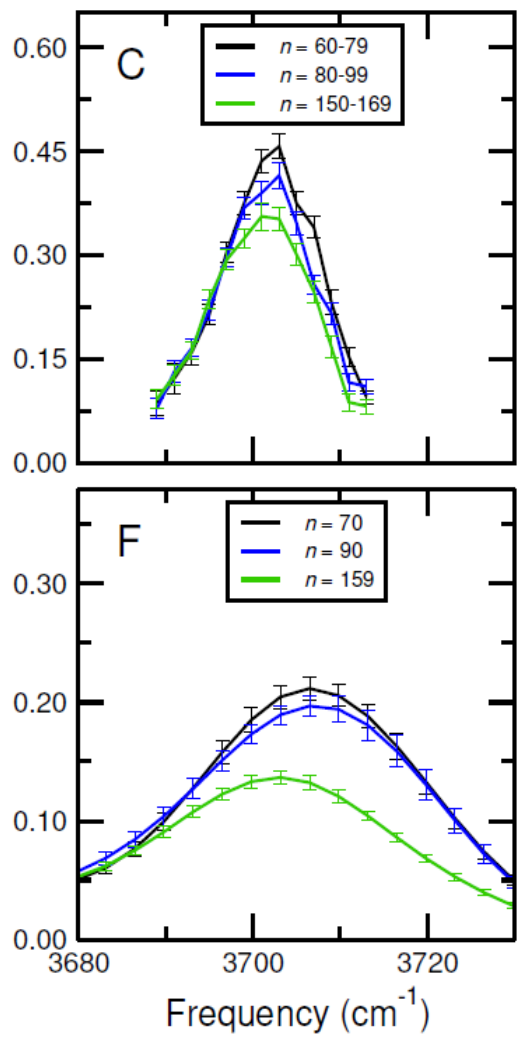

Experimental (upper panels A-C) and simulated (lower panels D-F) IR spectra of $\left(\mathrm{H}_{2} \mathrm{O}\right)_{n}$ clusters produced under slow cooling conditions showing the emerging peak at $3300 \mathrm{~cm}^{-1}$, a signature of crystallinity in the bonded $\mathrm{OH}$ stretch band at cluster sizes slightly below $n=100$ (panels $A$ and $D$ ). The $3700 \mathrm{~cm}^{-1}$ free $\mathrm{OH}$ stretch band also displays signs of crystallinity, namely the increase in intensity in the experimental spectrum, and the general redshift of the peak (panels $B$ and $E$ ). Note that all free $\mathrm{OH}$ band intensities are normalized against the integrated intensities of the bonded $\mathrm{OH}$ stretch band. Panels $\mathrm{C}$ and $\mathrm{F}$ show the contrasting size evolution of the free $\mathrm{OH}$ stretch band under fast cooling conditions producing solely amorphous clusters. The simulated spectra for $n=90$ and $n=159$ in panels $D$ and $E$ are weighted linear combinations of the IR spectra of amorphous and crystalline clusters (i.e. prepared with rapid cooling and slow cooling conditions, respectively). It is important to note that, particularly at smaller cluster sizes, the crystalline clusters are not purely crystalline, but are composed of a crystalline core with an amorphous surface layer. The error bars represent a 1 confidence interval. 


\section{Figure 3}

\section{$F_{\text {crystalline }}-F_{\text {amorphous }}$}

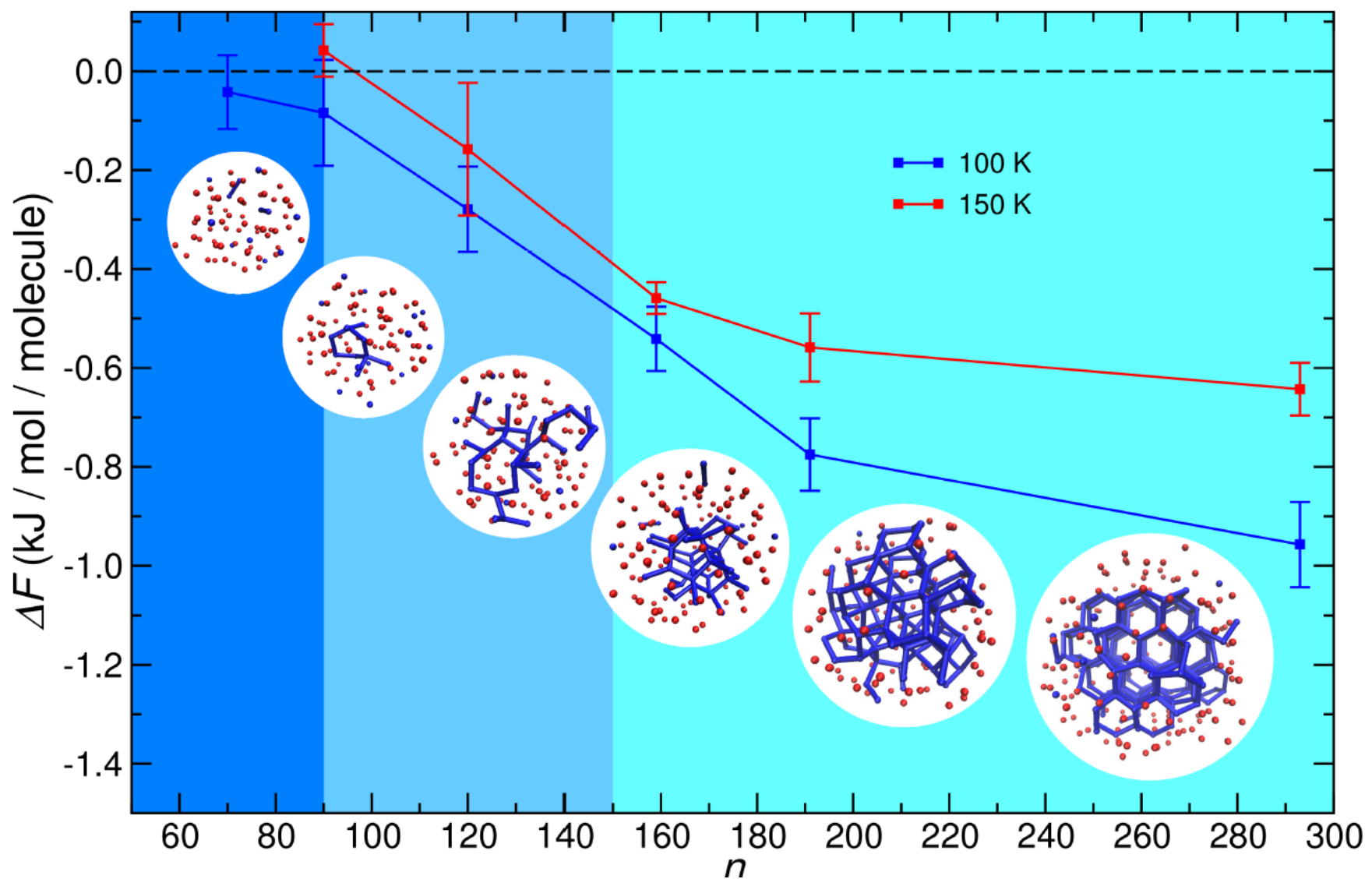

Simulated difference in molar free energy per molecule of amorphous and crystalline water clusters in the size range from $n=70$ to $n=293$. For $n=120$, initial structures were taken from Bandow and Hartke (ref. 5). For larger clusters, $\mathrm{mW}$ was used to nucleate clusters of varying sizes used as initial structures. Background shading indicates approximately the regions for which ice I is unstable (dark blue), amorphous and ice I clusters coexist through heterophasic oscillations in time (medium blue), and clusters crystallize to ice I through a well-defined first order transition (light blue). Snapshots of each simulated size of crystalline cluster are also included for comparison. 


\section{Supplementary Materials}

\section{Methods \\ Experimental set-up and IR spectra generation}

We have described the molecular beam generation and the subsequent experimental steps in the main text. Here we provide details on the laser systems and the generation of the IR action spectra shown in Figures 2 and S1 to S3. For the IR excitation of the clusters we used a tuneable IR OPO/OPA system (LaserVision) pumped by solid state laser (Continuum Powerlite 8000). Subsequent photionization was carried out with 80-100 ns delay at $385 \mathrm{~nm}$ with a solid state laser (Continuum Powerlite 9010) and pumped dye laser (Sirah Cobra Stretch). More details are found in ref. 22. Both lasers are operated at $10 \mathrm{~Hz}$ but the Q-switch of the IR laser only at $5 \mathrm{~Hz}$. Thus, per second, 5 mass scans with IR laser on and 5 scans with IR laser off are alternately recorded. The signal is IR induced relative ion signal gain. The complete IR spectra in Figures 2 and S1 were taken with $10 \mathrm{~cm}^{-1}$ step width for the bonded and $5 \mathrm{~cm}^{-1}$ around the free $\mathrm{OH}$ stretch bands. The free $\mathrm{OH}$ stretch spectra were taken with $2 \mathrm{~cm}^{-1}$ step width (Figures 2B, C, and S3). The bandwidth of the IR laser is $3.7 \mathrm{~cm}^{-1}$. The spectra in Figures 2A and S1 were averaged over three scans and smoothed by averaging three neighboring points (25\%, 50\%, 25\%) three times. The trends in the averaged spectra are seen in each individual scan and the onset becomes significant for the size range 80 to 99 (see Figure S1). To more precisely pinpoint the onset size we recorded the signal intensity with high averaging in the region of maximum intensity gain $\left(3180 \mathrm{~cm}^{-1}\right)$ and near the absorption maximum $\left(3420 \mathrm{~cm}^{-1}\right)$ for slow and fast cooling conditions. The results are shown and discussed in Figure S2. Also, the IR spectra for the narrow free $\mathrm{OH}$ stretch band under slow cooling conditions are recorded with much higher averaging resulting in the small error margins shown in Figure S3. We note that the Na influence on the IR spectra is negligible for the here analyzed size range and already limited for clusters containing only 20 water molecules [15].

\section{$\underline{\text { Simulation of aggregation and gas dynamics }}$}

The approximation of cluster temperatures and cooling rates for the present experiment is based on detailed simulations of the aggregation and gas dynamics with a hybrid Lagrangian-Eulerian approach of neon-water vapor mixtures expanding through the same microscale conical nozzle [21]. The simulations are accompanied by final temperature estimation with the energy balance model of Buck [13]. The mass difference of a factor of 2 between neon in the simulation and argon in the experiment leads to a slower beam, slightly reducing the cooling rates compared to the simulation. The simulations show that the nozzle geometry determines the shape of the cluster temperature profiles (and thus the cooling rates as a function of the distance from the nozzle entrance) while gas composition and stagnation pressure determine the final temperature. Variation of the latter shifts the temperature profiles parallel to the position axis [21]. The temperature gradients and cooling rates (derived from the simulated temperature gradients and cluster velocities) are highest near the nozzle entrance, they drop with the decreasing gas density and become very low $(<0.1 \mathrm{~K} / \mathrm{ns})$ when the clusters slowly approach their final temperatures. Therefore, very low cooling rates are achieved below $200 \mathrm{~K}$ for final temperatures of around $150 \mathrm{~K}$ according to the estimate of Buck's energy balance model. We note that final temperatures predicted by this model for different expansions are in very good agreement with the measured and simulated shifts in the relative populations of the cage and book isomer of 
$\left(\mathrm{H}_{2} \mathrm{O}\right)_{6}$ [13]. For the present argon experiment the estimated final temperature is around $150 \mathrm{~K}$ with is consistent with the trends from detailed simulations for neon seeded expansions at higher stagnation pressure. For the helium seeded expansion the estimated final temperatures are around $50 \mathrm{~K}$ drastically increasing the cooling rates above $150 \mathrm{~K}$.

\section{Preparation of amorphous and icy clusters with a parallel evolutionary algorithm}

The low lying critical onset size for crystallization of water clusters, experimentally determined in this study, was already predicted more than 10 years ago. Therefore, the applied methodology is described here in some detail. Evolutionary algorithms are a powerful method to solve global optimization problems. They mimic processes found in biological evolution like mutation, crossover and selection driving a population to consist of individuals that fit better to given boundary conditions. For water clusters the optimization task is the search for the global minimum structures in terms of total binding energy. The datasets representing the clusters by atomic coordinates or atomic coordinates and Euler angles are referred to as individuals. Amorphous and ice-like structures investigated in this article were obtained employing the PHENIX algorithm - a phenotype algorithm with niches. The operations as crossover and mutation are applied to the phenotype of the individuals which means coordinates and Euler angles rather than on bit level. When it comes to selection, individuals may occupy niches, which allows selection by additional characteristics instead of energy alone. The overall principle of the generation-based variant of the PHENIX algorithm shall be described below. A more detailed description can be found in refs. 39-41.

The initial populations of $n$ parent individuals may be created randomly, result from an earlier run, or consist of specially selected structures. Following the initialization phase, all $n(n+1)=2$ pairs of individuals including self-pairing enter the mating phase. Here crossover takes place. The crossover operator can be implemented such as both individuals are cut by the same arbitrarily chosen plane. The fragments then are crosswise reassembled into a complete individual. Additionally, mutation may occur meaning translational or orientational variation of water molecules. This is followed by a local optimization step employing a standard algorithm (conjugate gradient, quasi-Newton). The parent individuals are then appended to those generated during the mating phase. The subsequent selection takes into account the individuals' fitness (energy) and geometric features (niching) to guarantee diversity: Depending on the size, the water clusters exhibit a variety of motives of their $\mathrm{O}$ atom skeletal structure ranging from cubes, pentaprisms, and clathrate-like cages, to potato shaped structures. Given a fixed size $n$ a variety of isomers are created and for each isomer a number of orientational isomers with different $\mathrm{H}$ bond networks are possible. Their total binding energies extend over a certain range. These ranges overlap for different structural motives which is why selection by fitness alone is not the best choice. Having selected $n$ individuals, postprocessing starts by applying two additional operators on each individual. These are global orientational optimization and directed mutation. The further one is a full evolutionary algorithm used to find the best orientational isomer of the initial individual by acting on the spatial orientation of the molecules only. This is followed by a local optimization. The latter one spans a 3-dimensional grid over the cluster. Then a number of molecules contributing least to the total energy are picked and inserted with random orientation into the boxes of the grid. The energetically lowest conformation is taken for a local optimization. Finally, there are $n$ triples of individuals. From each of the $n$ triples of individuals the one with the lowest energy is selected to build up the new parental generation. 
Now the first generation is complete and the process is repeated iteratively starting with the next mating phase. It stops after a predefined number of generations or if the best energy has not changed over a given number of generations. Computing time needed increases drastically $\left(\right.$ almost $\mathrm{n}^{3}$ ) with cluster size. So PHENIX has been implemented as a master worker scheme in parallel using MPI [42] to take advantage of shared and distributed memory computers. However, the generation-based scheme described above forces all processes to synchronize at the end of the mating and the postprocessing phase which means idle time until the last worker has finished its portion of work successfully. In ref. 5, an asynchronous variant of the PHENIX algorithm has been introduced which is faster because it circumvents this disadvantage by renouncing successive generations. The operators applied to the individuals as well as the selection scheme remain the same as before. Both types of the PHENIX algorithm were used to create amorphous and icy clusters using the TTM2-F potential [32]. Amorphous samples were obtained by simply starting PHENIX with randomly generated clusters. As described in ref. 5, ice-like clusters have been prepared as spherical cutouts for the ice modifications Ic ([43]) and Ih ([44]), based on data from the Inorganic Crystal Structure Database (ICSD) [45]. After a global orientational optimization followed by a single local optimization, the interior partly preserves crystalline order. This was proven by superimposing the $\mathrm{O}$ atom skeletal structure of the result and the original structure. Molecules of the optimized structure being displaced less than a given threshold were considered being located consistently with positions in the original crystalline cutout. The procedure is described in elaborate detail in ref. 5. The findings furthermore suggested such optimized cutouts being energetically competitive with their amorphous counterparts beyond $n=90$. The globally orientational optimized cutouts of selected sizes finally were chosen as input for runs with the complete PHENIX algorithm with results that are distinctively lower in energy then those originating from a single local optimization described above. Crystalline order though is still partially preserved.

\section{Preparation of initial clusters with $\mathrm{mW}$}

Liquid water droplets of size $n=159,191$, and 293 water molecules were prepared and equilibrated for $1 \mathrm{~ns}$ at $298 \mathrm{~K}$. Before performing the cooling simulations of these water droplets, we estimate the freezing temperature of maximum crystallization rate for a droplet of radius $R$, $T_{\mathrm{f}}^{\text {max }}(R)=T_{\mathrm{f}}^{\text {bulk,max }}-K_{\mathrm{f}}(R-d)$, where $T_{\mathrm{f}}^{\text {bulk,max }}$ is the freezing temperature of bulk mW water, constant $K_{\mathrm{f}}=20 \mathrm{~K} \cdot \mathrm{nm}$, and $d=0.6 \mathrm{~nm}$ is a correction to the radius of the particle. Based on the estimated freezing temperature, 3 independent simulations for each droplet size were cooled in the temperature range from $T_{\mathrm{f}}^{\max }(R)+20$ and $T_{\mathrm{f}}^{\max }(R)-20$, with a cooling rate of $q^{\max }$ listed in Table S1. Each of these crystallized configurations were analyzed using CHILL+ [31], and the configuration with the highest fraction of ice I (hexagonal and cubic ice) was chosen to build the initial system for simulations with the MB-pol model. The cooling rate was chosen as to maintain thermal equilibrium (see ref. 6). All simulations of water nanodroplet crystallization using $\mathrm{mW}$ water were performed in LAMMPS [46]. The constant cooling simulations were performed in the NVT ensemble. The temperature was controlled with a Nosé-Hoover thermostat with relaxation time of 1 ps [47-49]. The equations of motion were integrated using the velocity Verlet algorithm and a $10 \mathrm{fs}$ timestep. 
Simulations with the atomistic polarizable MB-pol water molecule

Many-body molecular dynamics (MB-MD) simulations using the MB-pol potential energy function for water were performed to compute the infrared spectra of the ice clusters. These clusters were obtained either through $\mathrm{mW}$ simulations or PEA. To acquire more starting cluster configurations, as discussed in the main text, crystalline clusters were extracted from equilibrated ice $I_{h}$ bulk systems to create clusters of the desired size \pm 5 water molecules. Amorphous clusters were obtained by first melting existing crystalline clusters at $300 \mathrm{~K}$ in the $N V T$ ensemble for 100 ps before extremely rapidly freezing to the desired temperature.

Whether using experimental or $\mathrm{mW}$ derived initial configurations, the amorphous and crystalline clusters were equilibrated in the $N V T$ ensemble for 1000 ps. Following equilibration, $N V E$ simulations of 100 ps were performed. All MB-MD simulations using MB-pol were run with a modified version of the DL_POLY 2.0 molecular dynamics package [50]. Periodic boundary conditions were used in all cases with a box size of $100 \AA \times 100 \AA \times 100 \AA$. The velocity Verlet algorithm was used to integrate Newton's equations of motions with a time step of $0.2 \mathrm{fs}$. The 'always stable predictor corrector' (ASPC) method was used for the calculation of induced dipoles during the MB-MD simulations. Nosé-Hoover chains of four beads attached to each degree of freedom were used to control the temperature.

\section{Infrared Spectra}

The infrared spectra of all clusters were calculated with the many-body MB- $\mu$ dipole moment surface [51]. The vibrational spectra were calculated within the time correlation function formalism that relies on the following expressions. The IR activity is calculated as

$$
I_{I R}(\omega)=\left[\frac{2 \omega}{3 V \hbar c \epsilon_{0}}\right] \tanh (\beta \hbar \omega) \int_{-\infty}^{\infty} d t e^{-i \omega t}\langle\mu(0) \mu(t)\rangle
$$

with $V$ the system volume, $c$ the speed of light, $\epsilon_{0}$ the permittivity of free space, and $\beta=$ $(k T)^{-1}$, with $k$ the Boltzmann constant.

To create mixtures of crystalline and amorphous clusters to compare with experimental results, a linear combination was created of the spectra obtained from crystalline and amorphous clusters. In Figures 2D and 2E, for example, for the $n=90$ spectrum composed of $80 \%$ amorphous and $20 \%$ crystalline clusters, the spectra were added with factors of 0.8 and 0.2 , respectively, then the resulting mixed spectrum was renormalized.

\section{Helmholtz Free Energy Calculations}

For cluster sizes between $n=70$ and 191, individual configurations were extracted from the MBMD simulations. The number of configurations used ranged from 5 to 20, in order to obtain a calculated error in the free energy within $\pm 0.1 \mathrm{kcal} / \mathrm{mol}$. For $n=293$, only 2 configurations were used due to the cost of normal mode calculations. These configurations were optimized and the normal modes calculated through the diagonalization of the Hessian matrix. From the normal mode frequencies, the free energy of each cluster was approximated with the quasi-harmonic approximation [34-35],

$$
F=U_{c o n f}+\frac{1}{2} \sum_{i=1}^{n} h v_{i}+k_{B} T \sum_{i=1}^{n} \ln \left(1-e^{-\frac{h v_{i}}{k_{B} T}}\right)
$$


where $U_{\text {conf }}$ is the internal energy of the cluster configuration as calculated from DL_POLY 2.0, $n$ is the number of normal modes $v_{i}$ for the system, $h$ is Planck's constant, $k_{B}$ is Boltzmann's constant, and $T$ is the temperature of the system. The second term is the zero-point energy contribution and the last term is the vibrational contribution to the thermal excitation energy. 


\section{Bonded OH (Slow Cooling)}

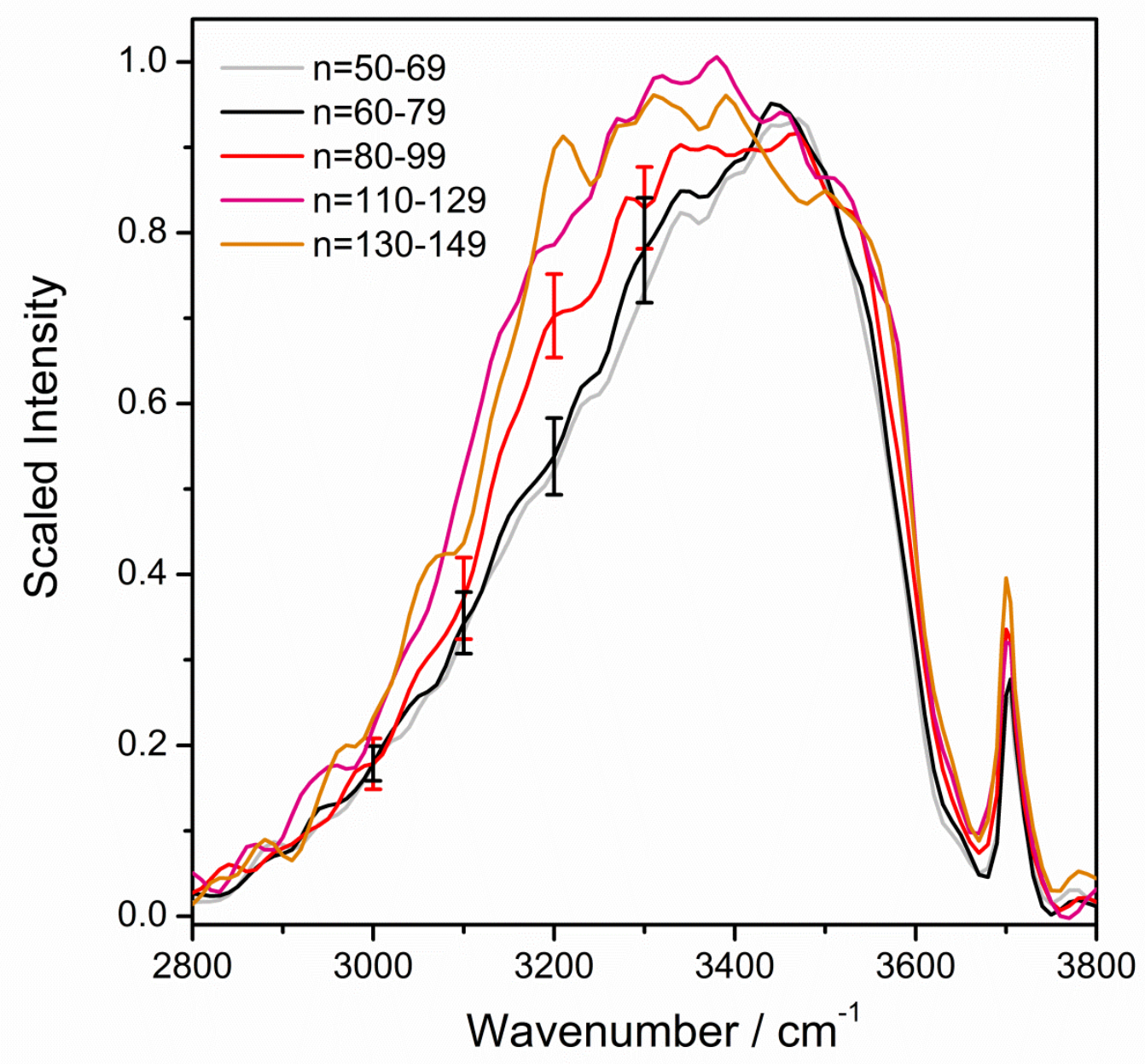

Fig. S1.

Detailed, size resolved analysis of the evolution of the bonded $\mathrm{OH}$ stretch spectra of $\mathrm{Na}\left(\mathrm{H}_{2} \mathrm{O}\right)_{\mathrm{n}}$ clusters in the onset region for crystallization under slow cooling conditions. The spectra belong to the experiment shown in Figure 2 and discussed in the main text. For noise reduction 20 cluster sizes are binned to each IR spectrum. The spectra show a sudden and significant (error bars belong to $1 \sigma$ confidence interval) intensity increase in the size slice from 80 to 99, clearly centered around $3200 \mathrm{~cm}^{-1}$, which we assign to the presence of $\mathrm{OH}$ oscillators in the ice I configuration, while the averaged spectra up to $n=79$ indicate purely amorphous clusters. Further we see a gradual intensity gain around $3200 \mathrm{~cm}^{-1}$ above $n=100$ and only minor changes in the spectral shape above $3400 \mathrm{~cm}^{-1}$, indicating a high fraction of clusters remaining in a purely liquid-like configuration. This transition region we assign to mixtures of ice I-like and purely amorphous clusters consistent with the IR spectra simulations and the free energy calculations shown in Figures 2 and 3 and discussed in the main text. 


\section{Bonded OH (Slow vs. Fast Cooling)}

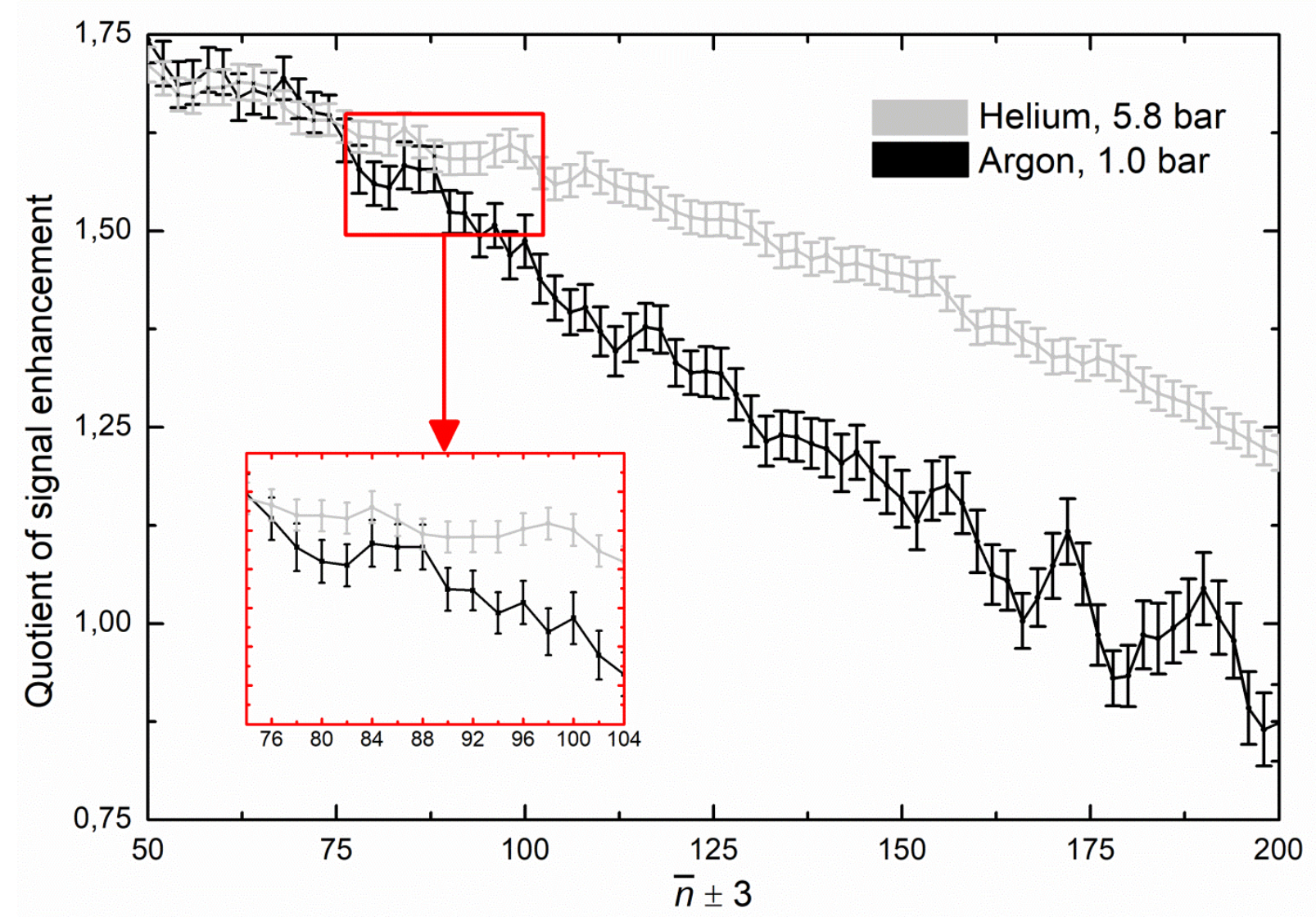

Fig. S2.

Size evolution of the signal intensity ratio of 3420 to $3180 \mathrm{~cm}^{-1}$ for slow and fast cooling conditions (helium seeded expansion at 5.8 bar stagnation pressure and a water mole fraction of 0.3). In both experiments seven cluster sizes are binned for each data point and the floating averages are shown. The error bars belong to $1 \sigma$. The inlet enlarges the traces in the onset region for crystallization. The traces start to clearly diverge around $n=90$, corresponding to the intensity gain around $3200 \mathrm{~cm}^{-1}$ only observed for slow cooling conditions and assigned to the emergence of water clusters with a core in ice I configuration. The inlet shows that $n=88 \pm 3$ is the last data point for which the error bars overlap, above $n=92 \pm 3$ the deviation of the traces is highly significant (better than $2 \sigma$ ). The first data points without overlapping error bars are at $n=80 \pm 3$ and $82 \pm 3$. The onset size region of $n=90 \pm 10$ reflects the results of this detailed analysis. 


\section{Free OH (Slow Cooling)}
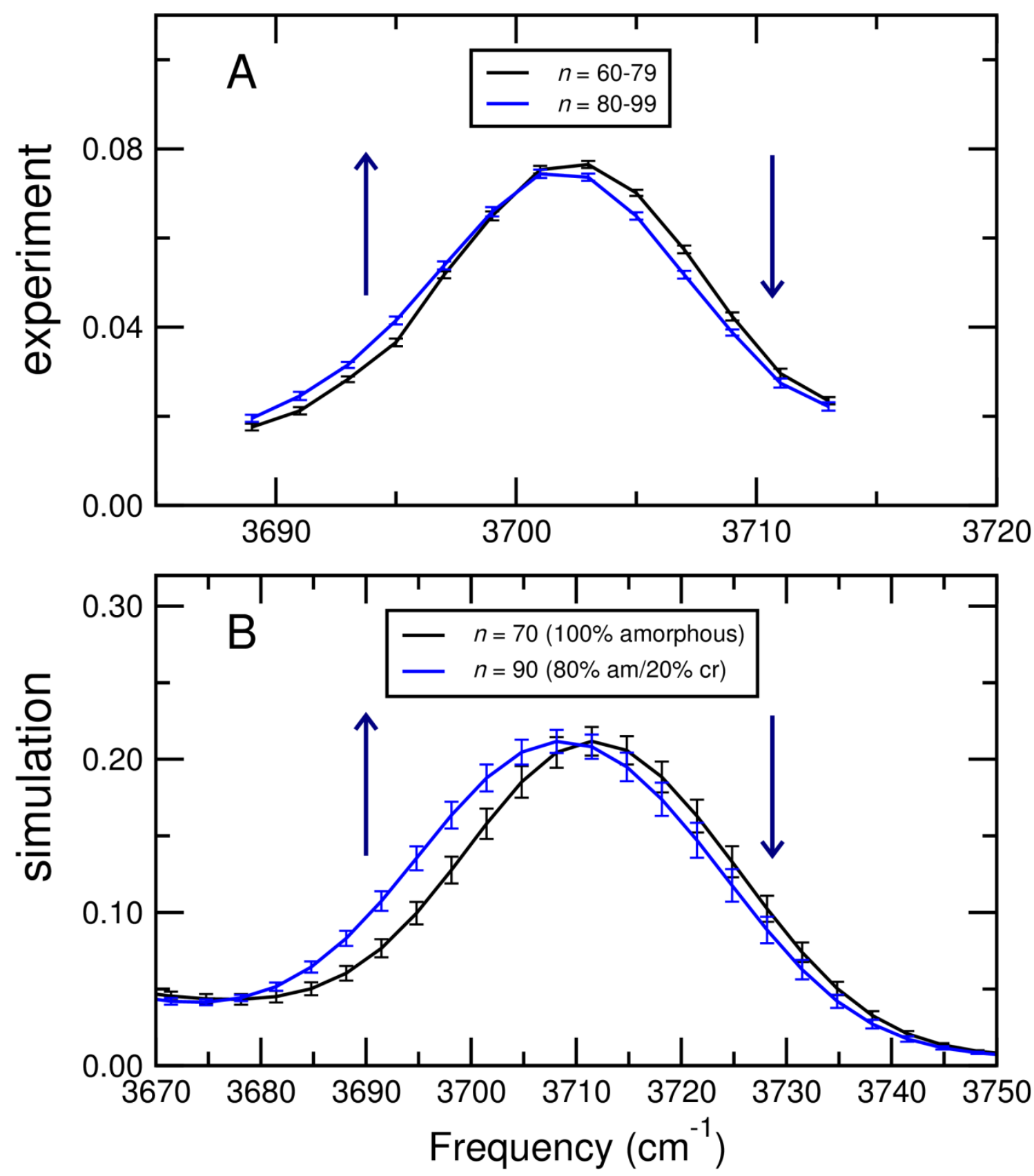

Fig. S3.

Enlarged views of the experimental (A) and simulated (B) free $\mathrm{OH}$ regions of the IR spectra of slow cooled clusters, reproduced from Figure 2. As mentioned in the main text, the shoulders on the red and blue sides increase and decrease, respectively, with increasing cluster size. This transition is a result of the shift to a more crystalline cluster, as replicated in the simulated spectra. Note that the axes in A and B are not equivalent. They have been scaled to highlight the similarity in overall shape and shifts between cluster sizes. 


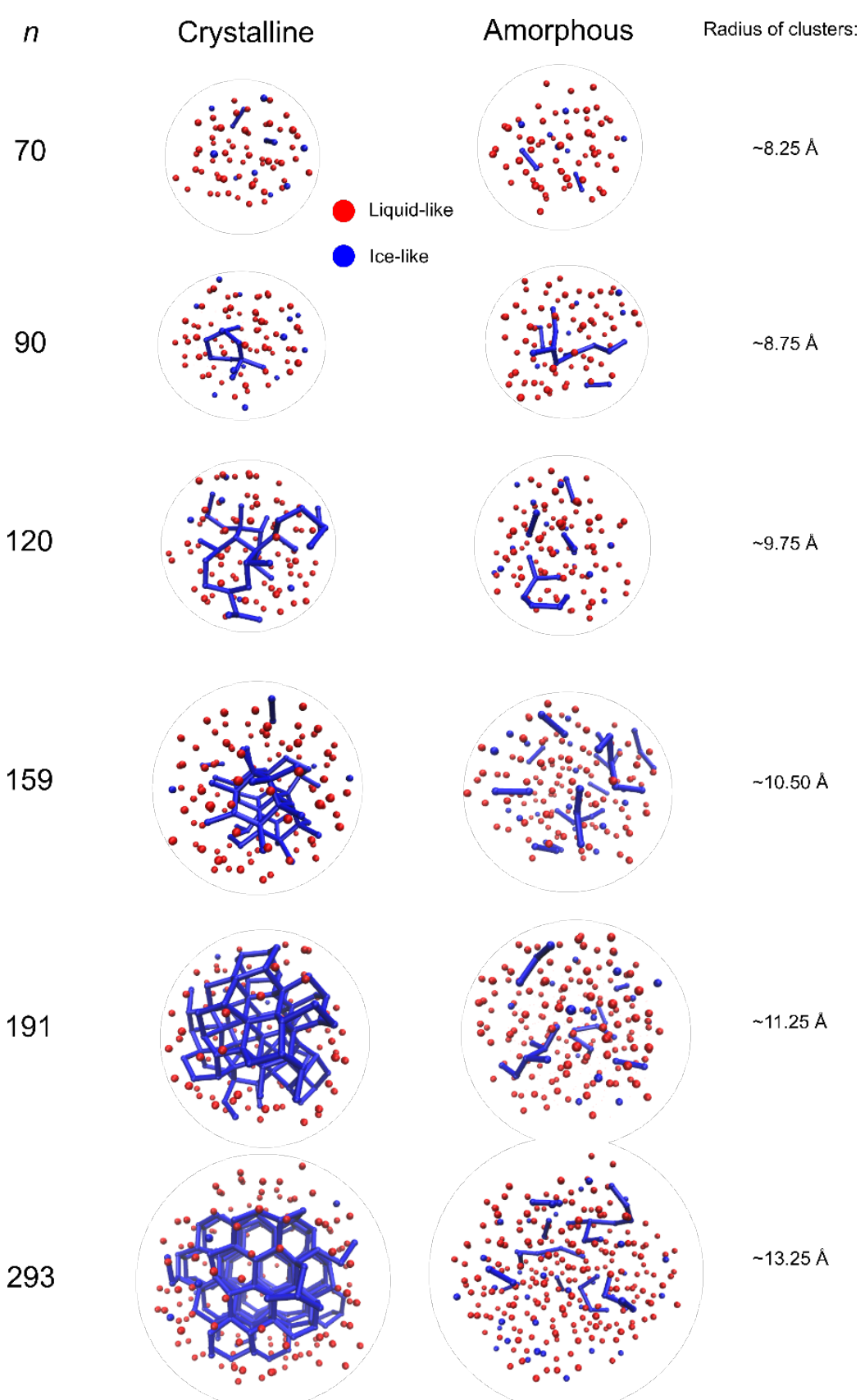

Fig. S4.

Snapshots from trajectories of amorphous and crystalline water clusters in the size range from $n=70$ to $n=293$. Clusters display only oxygen atoms of water molecules and are colored based on CHILL+ assignment as either liquid-like (red) or ice-like (blue). The crystalline core gradually becomes energetically unstable as size decreases from $n=293$ to $n=70$, while the amorphous clusters remain a mixture of liquid- and ice-like regions with no well-defined ice I core. Radii of clusters are obtained from sizes of regions extracted from bulk ice Ih structures that provide desired cluster sizes. The values for $n=159$ to 293 agree well with the radii approximated through $\mathrm{mW}$ simulations provided in Table S1. 


\begin{tabular}{|c|c|c|}
\hline $\boldsymbol{n}$ & $\boldsymbol{R}(\boldsymbol{\AA})^{\mathbf{b}}$ & $\mathbf{q}^{\mathbf{m a x}}\left(\mathbf{K ~ n s}^{\mathbf{- 1}}\right)$ \\
\hline 159 & 10.5 & $<0.05^{\mathbf{a}}$ \\
\hline 191 & 11.1 & 0.05 \\
\hline 293 & 12.9 & 0.10 \\
\hline
\end{tabular}

\section{Table S1.}

Radii and maximum cooling rates that produce ice for water nanodroplets generated from $\mathrm{mW}$ simulations for clusters of $n$ water molecules.

a. Configuration obtained from results of previous work, ref. 15.

b. We determine the radius $R$ of a water droplet from the molecular volume of ice in the mW model, $v=30.6$

$\AA^{3}$, and the number $n$ of water molecules in the particle, and assuming the particles are sphere, $R=(3 \mathrm{vn} / 4 \pi)^{1 / 3}$.

\section{References}

[39] B. Hartke, Global cluster geometry optimization by a phenotype algorithm with niches: location of elusive minima, and low-order scaling with cluster size. J. Comput. Chem. 20, 1752-1759 (1999).

[40] B. Hartke, Global geometry optimization of molecular clusters: TIP4P water. Z. Phys. Chem. 214, 1251-1264 (2000).

[41] B. Hartke, Size-dependent transition from all-surface to interior-molecule structures in pure neutral water clusters. Phys. Chem. Chem. Phys. 5, 275-284 (2003).

[42] The Message Passing Interface (MPI) standard: https://www.mcs.anl.gov/research/projects/mpi/index.htm

[43] G. P. Arnold, E. D. Finch, S. W. Rabideau, R. G. Wenzel, Neutron diffraction study of ice polymorphs. III. ice Ic. J. Chem. Phys. 49, 4365 (1968).

[44] S. W. Petersen, H. A. Levy, A single-crystal neutron diffraction study of heavy ice. Acta Cryst. 10, 70 (1957).

[45] Inorganic Crystal Struture Database (ICSD): https://icsd._z-karlsruhe.de/

[46] S. Plimpton, Fast parallel algorithms for short-range molecular dynamics. J. Comput. Phys. 117, 1-19 (1995)

[47] G. J. Martyna, M. L. Klein, M. Tuckerman, Nosé-Hoover chains: The canonical ensemble via continuous dynamics. J. Chem. Phys. 97, 2635-2643 (1992).

[48] S. Nosé, A unified formulation of the constant temperature molecular dynamics methods. J. Chem. Phys. 81, 511-519 (1984).

[49] W. G. Hoover, Canonical dynamics: Equilibrium phase-space distributions. Phys. Rev. A 31, 1695-1697 (1985).

[50] W. Smith, C. W. Yong and P. M. Rodger, DL_POLY: Application to molecular simulation. Mol. Sim. 28, 385-471 (2002).

[51] G. Medders, F. Paesani, Infrared and Raman spectroscopy of liquid water through "firstprinciples” many-body molecular dynamics. J. Chem. Theory Comput. 11, 1145-1154 (2015). 
